\title{
INTERVENCIÓN EDUCATIVA PARA EL CONTROL DEL DENGUE EN ENTORNOS FAMILIARES EN UNA COMUNIDAD DE COLOMBIA
}

\author{
Ena Escudero-Támara ${ }^{1,2, a}$, Gloria Villareal-Amaris ${ }^{1, b}$
}

\begin{abstract}
RESUMEN
Objetivos. Evaluar la eficacia de una intervención educativa para inducir cambios en la conducta de eliminar los criaderos del vector del dengue en familias de una comuna del Municipio de Sincelejo en Colombia. Materiales y métodos. Se realizó un estudio de una intervención educativa tipo antes y después, con un solo grupo a 54 familias seleccionadas por conveniencia. Se aplicó un programa educativo diseñado con los resultados de un test de conocimiento, una entrevista semiestructurada, guía de observación y los referentes del modelo de adopción de precauciones y aspectos de la teoría de comunicación para impactar en conducta (COMBI). El impacto fue medido mediante proporción de cambios y la prueba de McNemar. Resultados. Posintervención se lograron cambios en los niveles de conocimientos inadecuados sobre el dengue y comportamiento del vector de un $14,8 \%$ a un $3,7 \%(p=0,109)$, en sus creencias inadecuadas de un $20,4 \%$ a un $5,6 \%(p=0,008)$ y en la practicas adecuadas del 24 al $87 \%(p=0,001)$. Se logró que al final del proceso con cada uno de los grupos participantes clasificados el $64,8 \%$ se ubicaran en la etapa de acción. Se redujo el número de criaderos intradomiciliarios de Aedes, de un $92,6 \%$ a un $35,2 \%(p=0,001)$. Conclusiones. La intervención permitió conducir a los participantes a la adherencia de la conducta promovida. Fue útil subdividir a la población, en los que aplican las medidas preventivas y los que solo tienen la intención de realizarlas, porque permite al personal de salud adoptar las acciones de acuerdo a las características de cada grupo.
\end{abstract}

Palabras clave: Dengue/prevención \& control; Educación del paciente como asunto; Participación comunal (fuente: DeCS BIREME).

\section{EDUCATIONAL INTERVENTION FOR THE CONTROL OF DENGUE IN FAMILY ENVIRONMENTS IN A COMMUNITY IN COLOMBIA}

\begin{abstract}
Objectives. To evaluate the effectiveness of an educational intervention to induce changes in behavior to eliminate breeding places for the dengue vector in families from a community in the Municipality of Sincelejo, Colombia. Materials and methods. An educational intervention type was performed before and after, with a group of 54 families selected by convenience sampling. An educational program designed with the results of a knowledge test, a semi-structured interview, observation guide and the main concepts of the Communication for Behavioural Impact (COMBI) toolkit was applied. The impact was measured by proportion changes and the McNemar test. Results. Post-intervention changes were made in the levels of inadequate knowledge about dengue and behavior of the vector from $14.8 \%$ to $3.7 \%(p=0.109)$, in their inadequate beliefs from $20.4 \%$ to $5.6 \%(p=0.008)$ and in adequate practices from 24 to $87 \%(p=0.001)$. At the end of the process with each of the participant groups, $64.8 \%$ were classified in the action stage. There was a reduction of the number of intradomiciliary Aedes breeding places from $92.6 \%$ to $35.2 \%(p=0.001)$. Conclusions. The intervention lead participants to adhere to the behavior changes that were promoted. It was useful to subdivide the population into those who apply preventive measures and those who only have the intention of making them, because this enables the health personnel to adopt actions according to the characteristics of each group.
\end{abstract}

Key words: Dengue/prevention \& control; Patient education as topic; Consumer participation (source: MeSH NLM).

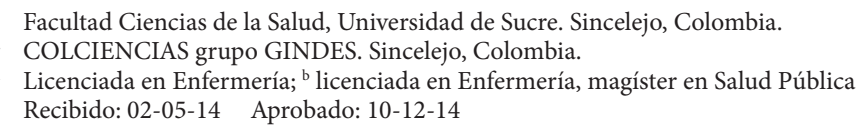

Licenciada en Enfermería; ${ }^{b}$ licenciada en Enfermería, magíster en Salud Pública Recibido: 02-05-14 Aprobado: 10-12-14

Citar como: Escudero-Támara E, Villareal-Amaris G. Intervención educativa para el control del dengue en entornos familiares en una comunidad de Colombia. Rev Peru Med Exp Salud Publica. 2015;32(1):19-25. 


\section{INTRODUCCIÓN}

El dengue es una enfermedad de interés en salud pública por su gran impacto en la morbilidad y mortalidad en el contexto mundial, regional y nacional. En Colombia, el dengue mantiene un comportamiento endémico como producto de la interacción de múltiples factores (1), estando de un lado los asociados a la conducta humana, entre las que se encuentra el almacenamiento del agua en forma inadecuada, conservar inservibles o recipientes a cielo abierto y la falta de compromiso de la comunidad en la prevención de la enfermedad (2).

Se han utilizado diferentes métodos de control vectorial como fumigaciones, avatización y las actividades educativas aún continúan teniendo un gran componente informativo sobre la enfermedad, pero no han sido eficaces porque no han logrado eliminar los criaderos del vector $y$, en consecuencia, minimizar el riesgo ${ }^{(3)}$.

El control del dengue requiere que las personas comprendan que sus estilos de vida hacen parte del problema. Por esto, las intervenciones sanitarias deben centrarse en las motivaciones, en sus comportamientos, en el seguimiento y control de las acciones, para garantizar su sostenibilidad. Además, asumir la educación en salud como un proceso, que permita diferenciar grupos con actitudes claramente distintas hacia la eliminación de criaderos del vector.

El objetivo del estudio fue evaluar la eficacia de un programa educativo para eliminar los criaderos del vector del dengue a la luz del modelo de adopción de precauciones y aspectos de la estrategia comunicación para impactar en conducta (COMBI) en un municipio de Sincelejo, Sucre en Colombia

\section{MATERIALES Y MÉTODOS}

\section{DISEÑO DE LA INVESTIGACIÓN}

Se realizó un estudio de intervención educativa de tipo antes y después, con un solo grupo siguiendo el diseño de Burns y Grove en el cual se mide un solo grupo de forma repetida, antes, durante y después de la exposición al tratamiento ${ }^{(4)}$.

El estudio, se realizó en la comuna 7 ubicada en la zona sur del Municipio de Sincelejo, Sucre en Colombia, conformada por 31 barrios con características de marginalidad y pobreza, y que cuenta con tres escuelas de básica primaria. En el momento del estudio la comuna presentó un índice aédico de $21 \%$; las escuelas resultaron positivas a la presencia del vector y una concentración de casos de dengue en tercer y cuarto grados, considerándose que los escolares se encontraban en riesgo, tanto en su entorno familiar como escolar. Se abordaron estudios en cada uno de estos entornos; en este artículo se presentan los resultados del abordaje familiar.

\section{POBLACIÓN DE ESTUDIO}

La población estuvo conformada por 80 familiares de escolares, seleccionados mediante la identificación de criaderos del vector en sus viviendas. Los escolares de tercero y cuarto grados de las tres escuelas de básica primaria públicas, resolvieron un cuestionario gráfico con los diferentes sitios posibles de criaderos en una vivienda, para que en él identificaran su vivienda. La presencia de criaderos positivos en las viviendas seleccionadas fueron confirmadas como positivas en un $92,2 \%$ (50) para formas inmaduras (larvas o pupas) de Aedes, por la Oficina de Salud Pública de la Secretaria de Salud Municipal, utilizando la dirección de la vivienda.

El propósito era identificar, desde los escolares, las viviendas con criaderos del vector y llegar a los padres o familiares; además, que los estimularan para una mejor respuesta a la intervención educativa propuesta.

Se trabajó con una muestra de 54 familiares de escolares que acudieron a la convocatoria y que reunieron los siguientes criterios de inclusión: lugar de residencia en la comuna de estudio, viviendas con uno o más criaderos del vector, aceptar voluntariamente participar en el estudio y la firma del consentimiento informado. La muestra se distribuyó en dos grupos de 27 familiares para iniciar el proceso de intervención, siguiendo el modelo para el estudio de patrones culturales de Romney et al. Los autores plantean, que el tamaño de la muestra no debe ser grande, debido a que la correlación promedio entre informantes tiende a ser alta. Weller y Romney (5) indican un tamaño mínimo de 17 informantes, si se espera clasificar el $95 \%$ de las preguntas correctamente, bajo un promedio de competencia cultural de $0,5 \mathrm{y}$ nivel de confianza de 0,95.

\section{DESCRIPCIÓN DE LA INTERVENCIÓN EDUCATIVA}

La estrategia educativa "Unidos contra el dengue: recoge los inservibles - lava y tapa los tanques" se basó en cuatro componentes como son: el objetivo, la conducta a promover, el proceso metodológico para la conducción de la adopción de la conducta basada en la teoría de adopción de precauciones y elementos de participación comunitaria de la estrategia COMBI y las metas; estos 
componentes se articularon con el propósito de motivar y estimular el cambio de conducta hacia la eliminación de los criaderos del vector del dengue.

Para implementar la estrategia educativa fue necesario levantar una línea de base para determinar los aspectos cognitivos y motivacionales de la conducta presente, frente a los comportamientos que se quieren cambiar. Estos resultados se confrontaron al final del proceso utilizándose los mismos instrumentos, para valorar los cambios alcanzados con la estrategia utilizada.

La estrategia se desarrolló concentrando geográficamente a los participantes en dos grupos y las acciones educativas se desarrollaron con mayor énfasis a nivel domiciliario y teniendo en cuenta la etapa de cambio en que estaba ubicado el participante, a través de técnicas de: conversatorios, lluvia de ideas, talleres demostrativos de lavado de recipientes recolectores de agua, de reflexión crítica apoyado con ayudas educativas: folletos, plegables, láminas, bocetos, mensajes educativos, juegos y programa radial. En la Tabla 1 se presenta un resumen de la estrategia educativa.

\section{RECOLECCIÓN Y ANÁLISIS DE LA INFORMACIÓN}

Para la recolección de la información se aplicó una encuesta al inicio de la intervención y a los seis meses después de la intervención. Se exploraron conocimientos sobre la enfermedad, comportamientos del vector y las medidas de prevención del dengue. Una entrevista semiestructurada antes y después, para explorar las creencias relacionadas con la percepción de riesgo de enfermar o morir y la severidad de la enfermedad. A través de una lista de chequeo se valoró la presencia de criaderos intradomiciliarios antes y después de la intervención y se monitorearon las conductas que contempla la estrategia educativa para determinar los cambios.

En el cuestionario, los ítems referentes a conocimientos y las creencias fueron valorados como ciertas o erróneas en la medida que estas fueran favorables o desfavorables para contraer la enfermedad. Las prácticas se controlaron calificando en la lista de chequeo como Sí aplica o No aplica en cada uno de los ítems que conformaba la conducta que promovió la estrategia educativa.

Las categorías se transformaron en adecuadas si el valor final de los ítems estaba por encima del $60 \%$ de total de ítems que conforman cada dimensión y, como inadecuada, si el valor total obtenido estaba por debajo.

En el caso de la dimensión "presencia de criaderos intradomiciliarios" se valoraron por el número de criaderos encontrados en cada una de las viviendas y se valoraron como viviendas con ausencia y presencia de criaderos del vector.

Los datos se tabularon utilizando la hoja de cálculo del programa Microsoft Excel 2010 y se exportaron a SPSS Windows versión 21.0, para su análisis. Se realizó la descripción de frecuencias y proporción de cambio de las dimensiones de la conducta entre la valoración inicial y final de la intervención. La medición de la efectividad del programa educativo y la comprobación de la prueba de hipótesis de cambio de comportamientos y la reducción de los criaderos, se determinó con la prueba de MacNemar con un nivel de significación de 0,05.

Tabla 1. Resumen de la estrategia educativa

\begin{tabular}{|c|c|}
\hline $\begin{array}{l}\text { Componente Educativo por } \\
\text { Etapas }\end{array}$ & Actividades \\
\hline 1. Ignora el problema & $\begin{array}{l}\text { Informar: talleres motivacionales para aumentar la confianza en la necesidad de aplicar las } \\
\text { medidas preventivas y el beneficio familiar de aplicar las medidas de control del vector. }\end{array}$ \\
\hline $\begin{array}{l}\text { 2. Reconoce el problema } \\
\text { 3. Reconocimiento de la propia } \\
\text { susceptibilidad }\end{array}$ & $\begin{array}{l}\text { Talleres de motivación y cognitivos para sensibilizar la necesidad de asumir una conducta } \\
\text { favorecedora, focalizar las falencias cognitivas sobre la enfermedad y destacar los riesgos que } \\
\text { acarrea una actitud favorecedora de criaderos de mosquitos. Además, reforzar en el beneficio de } \\
\text { aplicar las medidas de eliminar los criaderos. } \\
\text { Se invita a la acción: se entrega y visualiza el slogan del programa educativo. }\end{array}$ \\
\hline $\begin{array}{l}\text { 4. Decide qué hacer } \\
\text { 5. Decide actuar }\end{array}$ & $\begin{array}{l}\text { Conversatorios para estimular la acción, plan de acción para comenzar las actividades - motivar } \\
\text { y discutir los beneficios. Entrega de folletos, plegables y dejar el juego Gánale al dengue- visitas } \\
\text { periódicas. }\end{array}$ \\
\hline $\begin{array}{l}\text { 6. Acción } \\
\text { 7. Mantenimiento. }\end{array}$ & $\begin{array}{l}\text { Talleres de demostración para hacer retroalimentación conjunta con otros participantes. } \\
\text { Realización de acciones colectivas. }\end{array}$ \\
\hline $\begin{array}{l}\text { Actividades comunicativas y } \\
\text { de participación. (dirigidas a } \\
\text { todos los participantes de las } \\
\text { diferentes etapas. }\end{array}$ & $\begin{array}{l}\text { Jornada educativa: Informando al vecino: compromiso de a tres vecinos cercanos educarlos sobre } \\
\text { la forma de prevenir la enfermedad y las medidas preventivas aprendidas. Utilizaron un juego } \\
\text { didáctico Gánale al dengue, periódicos murales, plegables, maquetas alusivas a la transmisión } \\
\text { del dengue, láminas consecuencias de la enfermedad. } \\
\text { Jornada de recolección de inservibles } \\
\text { Jornada de lava y tapa tus tanques. } \\
\text { Programa radial: Contra el zancudo por tu salud. }\end{array}$ \\
\hline
\end{tabular}




\section{CONSIDERACIONES ÉTICAS}

El protocolo de la investigación fue aprobado por el Comité de Ética en Investigación de la Universidad de Sucre, en la categoría de investigación sin riesgo, según la Resolución 8430 de 1993 del Ministerio de Salud de Colombia. Todos los participantes fueron debidamente informados del alcance del estudio, decidiendo su participación de modo voluntario y firmando el consentimiento informado.

\section{RESULTADOS}

De los 54 familiares de escolares, el $88,9 \%$ (48) correspondió al sexo femenino, con un rango de edad entre 23 y 48 años con un promedio de $36,19 \pm 12,49$. El $42,6 \%$ tienen un nivel educativo adecuado, el $74,1 \%$ (40) alcanzaron niveles de primaria y secundaria completa.

La Tabla 2 muestra los principales factores de riesgos en la línea de base identificados a nivel de la vivienda del escolar que fundamentó la estrategia educativa de intervención. Es muy alta la percepción de la presencia de zancudos $(70,4 \%)$ como la proporción de viviendas con criaderos positivos (93\%) y el $88 \%$ de ellas se encontraron positivas para criaderos de Aedes. En las viviendas en las cuales la familia no percibe zancudos, también se hallaron criaderos positivos.

Tabla 2. Línea de base de distribución de factores de riesgo según presencia de criaderos intradomiciliarios positivos

\begin{tabular}{|c|c|c|c|c|}
\hline \multirow[b]{2}{*}{ Factores de riesgo } & \multicolumn{2}{|c|}{ Viviendas } & \multicolumn{2}{|c|}{$\begin{array}{c}\text { Presencia de } \\
\text { criaderos positivos* }^{*}\end{array}$} \\
\hline & $\mathbf{n}$ & $(\%)$ & Sí & $(\%)$ \\
\hline \multicolumn{5}{|c|}{ Conocimientos sobre la enfermedad } \\
\hline Adecuado & 46 & $(85,2)$ & 35 & $(70)$ \\
\hline Inadecuado & 8 & $(15,0)$ & 6 & $(11)$ \\
\hline \multicolumn{5}{|c|}{$\begin{array}{l}\text { Percepción sobre la severidad de la enfermedad } \\
\text { de las personas }\end{array}$} \\
\hline Adecuada & 43 & $(80,0)$ & 41 & $(82)$ \\
\hline Inadecuada & 11 & $(20,0)$ & 9 & (18) \\
\hline \multicolumn{5}{|l|}{ Prácticas } \\
\hline Adecuadas & 13 & $(24,0)$ & 9 & $(18)$ \\
\hline Inadecuadas & 41 & $(76,0)$ & 41 & (82) \\
\hline \multicolumn{5}{|c|}{ Presencia de zancudos } \\
\hline Sí & 48 & $(70,4)$ & 44 & $(88)$ \\
\hline No & 6 & $(29,6)$ & 5 & (10) \\
\hline $\begin{array}{l}\text { Situación de vivienda } \\
\text { con criaderos }\end{array}$ & 50 & $(93,0)$ & 44 & (88) \\
\hline
\end{tabular}

* Información suministrada por Secretaria de Salud Municipal
El nivel de conocimientos de los encuestados se consideró adecuado en un $85,2 \%$, quienes conocen que la enfermedad se produce por un zancudo o mosquito, aunque se les dificulta pronunciar su nombre, saben dónde se crían, resaltan a los tanques o albercas donde recolectan el agua para consumo como el mayor criadero e identifican los síntomas de la enfermedad. Sin embargo, en el $70 \%$ de sus viviendas se observaron criaderos positivos como también en los que resultaron con conocimientos inadecuados, en un $11 \%$.

Entre los conocimientos inadecuados atribuidos a la enfermedad del dengue, el $14,8 \%$, señala a las aguas negras, los arroyos, los lotes enmontados y a la falta de fumigaciones. Los síntomas lo asociaron con procesos gripales y dolor en los huesos.

Con respecto a las creencias sobre la enfermedad, la susceptibilidad de enfermarse fue percibida en una forma adecuada en el $79,6 \%$, la población encuestada estuvo de acuerdo en permitir la revisión de las viviendas, desechar lo inservible, con la no eliminación del abate en sus depósitos de agua porque son en beneficio de la salud de su familia y que las personas ayudan a que la enfermedad no se pueda controlar. También reconocen que es una enfermedad que le puede atacar a cualquier persona; sin embargo, el $20 \%$, percibe como innecesarias las acciones realizadas por el programa de control de vectores, sobre todo por la aplicación de productos químicos o la abatización del agua.

Con respecto a la percepción de gravedad de la enfermedad, sus creencias también fueron adecuadas o correctas, se encontró que el grupo de personas encuestadas concuerdan que es una enfermedad grave, peligrosa y que causa la muerte.

En cuanto a las prácticas se consideraron inadecuadas en el $76 \%$ de los encuestados a pesar de asegurar aplicar medidas preventivas y de control de la enfermedad, se observó diferencias entre lo manifestado y la acción. El $44 \%$ de estas viviendas fueron positivas para criaderos de Aedes.

En la Tabla 3, se muestra que después de la intervención educativa el nivel de conocimientos de los participantes se incrementó de un 85,2 a un $96,3 \%$, obteniéndose una proporción de cambio del $75 \%$, sin embargo, esta diferencia no fue significativa $(p=0,109)$. El nivel de creencias se incrementó del 79,6 al $99,4 \%$, con un incremento de cambio del $73 \%(p=0,008)$ de igual manera, en las prácticas inadecuadas se registra un incremento de 24 a $87 \%$ para una proporción de cambio de $83 \%(p=0,001)$. 
Tabla 3. Nivel de conocimientos, creencias, prácticas antes y después de la intervención

\begin{tabular}{|c|c|c|c|c|c|}
\hline & \multicolumn{2}{|c|}{ Antes } & \multicolumn{2}{|c|}{ Después } & \multirow[b]{2}{*}{ Valor $p$} \\
\hline & $\mathbf{N}$ & $(\%)$ & $\mathbf{N}$ & $(\%)$ & \\
\hline \multicolumn{6}{|c|}{ Conocimientos } \\
\hline Inadecuados & 8 & $(14,8)$ & 2 & $(3,7)$ & 0,109 \\
\hline Adecuados & 46 & $(85,2)$ & 52 & $(96,3)$ & \\
\hline \multicolumn{6}{|l|}{ Creencias } \\
\hline Inadecuadas & 11 & $(20,4)$ & 3 & $(5,6)$ & 0,008 \\
\hline Adecuadas & 43 & $(79,6)$ & 51 & $(94,4)$ & \\
\hline \multicolumn{6}{|l|}{ Prácticas } \\
\hline Inadecuadas & 41 & (76) & 7 & (13) & 0,001 \\
\hline Adecuadas & 13 & (24) & 47 & (87) & \\
\hline
\end{tabular}

Se obtuvo una reducción en la presencia de criaderos en las viviendas de 92,6 a $35,2 \%$ con un porcentaje de cambio global en los diferentes tipos de criaderos de $53 \%(p=0,001)$. Después de la intervención educativa se encontró que los recipientes con agua de consumo sin tapas obtuvieron una proporción de cambio del $55 \%$, las botellas y recipientes dispersos en el patio un $61 \%$, canecas de basuras sin tapas un $44 \%$ (Tabla 4 ).

La clasificación de los participantes del programa educativo, según las etapas de cambio de la teoría de adopción de precauciones, se muestra en la Figura 1. Antes de la intervención un gran porcentaje de las familias que toma la decisión de adoptar el comportamiento protector, pero no lo pone en práctica $(29,5 \%)$ se le ubicó en la etapa 5 del proceso de cambio. El grupo que decide empezar el programa educativo y poner en práctica las medidas preventivas, se clasifica en la etapa 6 de la acción, correspondiente al 25,9\%, y un grupo de familias que aplicaba las medidas, no

Tabla 4. Presencia y tipos de criaderos antes y después de la intervención

\begin{tabular}{|c|c|c|c|c|c|}
\hline \multirow[b]{2}{*}{ Presencia criaderos } & \multicolumn{2}{|c|}{ Antes } & \multicolumn{2}{|c|}{ Después } & \multirow{2}{*}{$\begin{array}{l}\text { Proporción } \\
\text { de cambio } \\
(\%)\end{array}$} \\
\hline & $\mathrm{n}$ & (\%) & $\mathbf{n}$ & (\%) & \\
\hline \multicolumn{6}{|l|}{ Criaderos } \\
\hline Ausencia & 4 & $(7,4)$ & 35 & $(64,8)$ & $62 \%$ \\
\hline Presencia & 50 & $(92,6)$ & 19 & $(35,2)$ & \\
\hline \multicolumn{6}{|l|}{ Tipos de criaderos } \\
\hline $\begin{array}{l}\text { Recipientes con agua } \\
\text { de consumo sin tapas }\end{array}$ & 49 & $(48)$ & 22 & $(46)$ & $55 \%$ \\
\hline $\begin{array}{l}\text { Canecas de basuras } \\
\text { sin tapas }\end{array}$ & 18 & $(17)$ & 10 & $(21)$ & $44 \%$ \\
\hline $\begin{array}{l}\text { Botellas y recipientes } \\
\text { dispersos en el patio }\end{array}$ & 31 & $(30)$ & 12 & (25) & $61 \%$ \\
\hline Llantas con agua & 5 & $(5)$ & 4 & $(8)$ & $20 \%$ \\
\hline Total & 103 & $(100)$ & 48 & $(100)$ & $53 \%$ \\
\hline
\end{tabular}

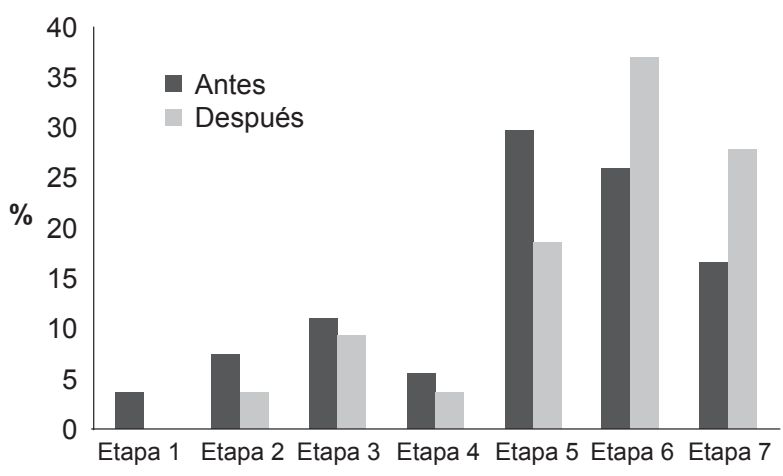

Figura 1. Etapas del modelo de adopción de precaución antes y después de la intervención

se hallaron criaderos (4) pero aún tenían deficiencias se les clasificó en la etapa de mantenimiento (etapa 7) correspondiente al $16,7 \%$. En las etapas de menor motivación se clasificaron a las familias que reconocieron su propia susceptibilidad frente a la enfermedad, pero no hay compromiso de acción (etapa 3 ) un 11,1 \%, en la etapa que reconoce el problema, pero son indiferentes a la acción (etapa 2) un 7,4\% y un $3,7 \%$ que ignoró el problema y con indiferencia a la acción, se clasificó en la etapa 1. Finalizada la intervención, el $64,8 \%$ (35) de los participantes terminaron en la etapa 6 y en la etapa 7 un $18,5 \%$ (10) inició la etapa 3.

\section{DISCUSIÓN}

Los resultados del presente estudio evidencian la efectividad de la intervención educativa realizada mediante el modelo de adopción de precauciones y aspectos de la estrategia comunicativa $\mathrm{COMBI}$, por cuanto se lograron movilizaciones de los cuidadores de los escolares en las diferentes etapas de cambio de comportamiento, los cuales fueron significativos en los distintos aspectos que determinan la conducta para la eliminación de los criaderos del vector.

El análisis muestra que los participantes de la intervención, en su inicio, se ubicaron en las primeras etapas que plantea el modelo de la adopción de precauciones y que existían barreras para llevar a cabo las conductas saludables. El brindar información centrada en incrementar la percepción del riesgo y del grado de vulnerabilidad que representa la enfermedad, a nivel individual, familiar y en la comunidad, permitió el impulso para tomar la decisión de iniciar en algunas y el avance, en otras, hacia la adopción de la conducta que promocionó la estrategia educativa. Lo anterior reafirma que la adopción de la precaución en salud es un proceso discontinuo que transcurre por diversas 
etapas, y que estas constituyen una sucesión obligada por la que debe transcurrir la persona en camino hacia la adopción de la precaución ${ }^{(6)}$.

Otro aspecto importante y decisivo en los resultados obtenidos fue el enmarcar la acción educativa como un proceso, en el control y monitoreo permanente de comportamientos de los participantes y el delegar acciones y establecer compromisos, en cada una de los grupos clasificados en las distintas etapas que predice el modelo de adopción de precauciones. Lo anterior, fundamentado en que algunos comportamientos son difíciles de modificar porque no son fácilmente controlables y se requiere de apoyo para afrontar dificultades y recaídas, que finalmente resultan en el control del comportamiento ${ }^{(7)}$.

La motivación de las personas para adoptar nuevas conductas preventivas fue decisiva y determinante en los cambios de comportamientos y en la movilización por las etapas de cambio, estos resultados son coincidentes con autores como Florez y Carranza ${ }^{(6)}$, Rodríguez y Londoño ${ }^{(8)}$, quienes reportan que en la mayor proporción de participantes de su estudio, aproximadamente las dos terceras partes $(70 \%)$, se ubican en etapas motivacionales inferiores a la acción, es decir, por debajo de la etapa seis. La clasificación de los sujetos en las diversas etapas motivacionales que propone la teoría de adopción de precauciones pone de manifiesto que se deben realizar acciones diversas para cada etapa de cambio en la que se ubiquen los participantes.

La etapa motivacional, en consecuencia, es una medición válida y confiable de una variable adecuada para planificar los procedimientos a seguir durante la intervención y para evaluar su impacto. La sola información, procedimiento tradicional de prevención y de promoción en las acciones que impulsan en Colombia las instituciones promotoras de salud, constituye una forma de intervención bastante limitada en sus alcances que puede beneficiar solamente a un grupo minoritario de la población ${ }^{(9)}$.

Diversos autores han observado que un adecuado nivel de conocimiento no necesariamente conduce a la realización de buenas prácticas ${ }^{(10-15)}$. Esto se confirma en la presente investigación en la medida que los cuidadores presentaron niveles de conocimientos adecuados y bajas prácticas en las diferentes etapas antes de la intervención, pero se incrementaron una vez finalizada. Se confirmaron los supuestos teóricos del modelo que plantea que cognoscitivamente la posición frente a la conducta preventiva depende del conocimiento, de las actitudes o de la motivación de la persona frente al comportamiento específico o acción de que se trate, y conductualmente, la posición puede ser de ausencia de práctica, práctica, o mantenimiento de la acción ${ }^{(6)}$.

En futuras investigaciones, es necesario incluir la valoración del índice aédico posintervención, que confirme la presencia del vector en los criaderos residuales para potenciar la efectividad de la intervención educativa, además, ampliar el tiempo de intervención, que permitió seguir valorando las familias que quedaron en la etapa que iniciaron la acción.

La eficacia de la estrategia educativa se logra al aplicar un proceso educativo diseñado y planeado para fortalecer y reforzar el nivel cognitivo y conductual de los participantes y trabajar sobre las barreras que impiden que las personas no adquieran el hábito saludable, a pesar del nivel de conocimiento e información que poseen. Para lograr cambios en la adopción de prácticas favorables y controlar la aparición del dengue, se deben diversificar las actividades educativas acorde con la necesidad y la etapa de cambio de la persona, considerando aspectos como los factores de riesgo existentes, el nivel de motivación y la disposición para cambiar, y el monitoreo permanente para que los programas educativos sean exitosos.

En conclusión, los hallazgos obtenidos en este estudio evidencian la efectividad de la estrategia educativa implementada, logrando que un alto porcentaje de los participantes adoptaran las conducta de lavar y tapar los tanques o recipientes de almacenamiento de agua y recogiendo los inservibles en sus viviendas, siguiendo el protocolo de la Secretaría de Salud Pública Municipal durante el tiempo de monitoreo y control.

Agradecimientos: a la secretaría de Salud Municipal de Sincelejo por el suministro de la información de la situación epidemiológica de la enfermedad y apoyo en la valoración entomológica de la zona de trabajo,

Contribuciones de los autores: EET participó en la concepción y diseño del estudio, obtención de resultados, análisis e interpretación de datos y la redacción, además obtuvo el financiamiento. GVA, brindó asesoría técnica, realizó la revisión crítica y la aprobación de la versión final artículo.

Fuentes de financiamiento: investigación realizada como beca pasantía en la modalidad tradicional, en el marco del Programa Jóvenes Investigadores e Innovadores de COLCIENCIAS (Colombia) bajo la convocatoria 566 de 2012.

Conflictos de interés: el autor manifiesta ser acreedor de la beca pasantía en la modalidad tradicional, en el marco del Programa Jóvenes Investigadores e Innovadores de COLCIENCIAS. 


\section{REFERENCIAS BIBLIOGRÁFICAS}

1. Maestre-Serrano R, Gómez-Camargo D. Dengue: Epidemiología, políticas públicas y resistencia de vectores e insecticidas. Rev Cienc Biomed. 2013;4(2):302-17.

2. Londoño AM, Molano LC, Rojo RA. Evaluación de la Estrategia "Maratón contra el dengue” y la percepción que sobre la enfermedad tiene la población escolar de la comuna 2 de Medellín en el año 2008. Medellín: Universidad CES; 2009.

3. Vesga-Gómez C, Cáceres-Manrique $\mathrm{F}$ de M. Eficacia de la educación lúdica en la prevención del Dengue en escolares. Rev Salud Publica. 2010;12 (4):55869.

4. Sousa V, Driessnack M, Costa I.Revisión de diseños de investigación resaltantes para Enfermería. Parte 1: diseños de investigación cuantitativa. Rev Lat- Am Enferm. 2007;15(3):5027.

5. Caballero R, Villaseñor A. Conocimientos sobre VIH/SIDA en adolescentes urbanos: consenso cultural de dudas e incertidumbres. Salud Publica Mex. 2003:45(Supl 1):S108-14.

6. Flórez L. El proceso de adopción de precauciones en la promoción de la salud. Rev Argent Clin Psic. 2002; 11(1):23-33.

7. Landeros E, Benavides R, Frederickson K, Martínez M, Celis M. Análisis de concepto:" decisión de cambio" para mejorar las conductas de salud en el contexto de las enfermedades crónicas en adultos. Aquichan. 2013;13(1):57-68.

8. Rodríguez I, Londoño C. El Proceso de adopción de precauciones en la prevención secundaria del consumo de cigarrillo en estudiantes universitarios. Act Colom Psicol. 2010;13(1):79-90.

9. Rodríguez I, Londoño C. El Proceso de adopción de precauciones en la prevención secundaria del consumo de cigarrillo en estudiantes universitarios. Acta Colomb Psicol. 2010;13(1):7990.

10. González A, Ibarra A. Nivel de conocimientos, actitudes y prácticas sobre la prevención del mosquito Aedes aegypti en comunidades del municipio Diez de Octubre, La Habana. Rev Cubana Hig Epidemiol. 2011;49(2):247-59.

11. Márquez $\mathrm{B}$, Borges $\mathrm{R}$, Rodríguez $\mathrm{M}$. Evaluación de conocimientos sobre el dengue en escolares de primera etapa de la Parroquia Coche, Caracas, Venezuela. Bol Mal Salud Amb. 2007;47(2):205-12.
12. Ávila GA, Araujo R, Leontsini E, Orellana G, Fernández E. Un programa escolar para el control del dengue en Honduras: del conocimiento a la práctica. Rev Panam Salud Publica. 2012;31(6):518-22.

13. Alcalá L, Hernández A. Conocimientos, actitudes y prácticas frente a la prevención del dengue, en estudiantes del grado 6-1 de una institución educativa en Ibagué-Tolima. Bio-grafía: Escritos sobre la Biología y su Enseñanza 2012;5(8):9-31.

14. Benitez-Leite S, Machi ML, Gibert E, Rivarola K. Conocimientos, actitudes y prácticas acerca del dengue en un barrio de Asunción. Arch Pediatr Urug. 2002;73(3):171-8.

15. Cáceres-Manrique FM. Vesga-Gómez C, Perea-Florez X, Ruitort M, Talbot Y. Conocimientos, actitudes y prácticas sobre dengue en dos barrios de Bucaramanga, Colombia. Rev Salud Publica. 2009;11(1):27-38.

Correspondencia: Ena Escudero Támara Dirección: Diagonal 26 No. 26-46 La Maria. Sincelejo, Sucre, Colombia Teléfono: (575) 2762742

Correo electrónico: enaescudero@gmail.com 